\title{
Theoretical Model for the Effect of Temperature on the Fluorescence Spectrum of Laser Dye (Rh6G) in Acetone
}

\author{
Mahasin F. Hadi Al-Kadhemy*, Ali A. Dawood Al-Zuky, Halah Fakhir Daear \\ Department of Physics, College of Science, Al-Mustansiriyah University, Baghdad, Iraq \\ *E-mail address: drmahasinf@yahoo.com
}

\begin{abstract}
In this research, the effect of temperature change on the fluorescence intensity for Rhodamine 6G $(\mathrm{Rh} 6 \mathrm{G})$ in acetone from room temperature to freezing point of solvent by using liquid nitrogen was studied experimentally and theoretically. As we have noted that the increased temperature led to less intensity and increasingly broader spectrum. We estimate theoretical model for this effect, and the theoretical equation was Extra Val equation.
\end{abstract}

Keywords: Laser Dye; Rhodamine 6G; Fluorescence Spectrum; Effect of Temperature; Theoretical Model

\section{INTRODUCTION}

Organic dyes of xanthene family have been extensively used as an active medium for tunable dye laser with tunability range of wavelengths. One of the most commonly used xanthene dye is Rhodamine 6G (R6G) emitting in the red region of visible spectrum. This dye is also used as sensor [1], non-linear optical material [2], photo sensitizer [3], and malarial protease labels [4]. The effect of temperature on the fluorescence spectra of organic laser dye solutions has received considerable attention [5-7]. M. A. Ali et al [8] studied the effect of temperature on an active medium Rhodamine B in ethylene glycol of dye laser and the results noticed that there is significant improvements in the performance of this dye laser were achieved as the solvent temperature was reduced below room temperature, namely: a significant reduction in the threshold power requirements and a considerable increase in the attainable output power. The results obtained may be understood in terms of increased quantum yield of the RhB dye as the temperature is reduced. Mahasin F. Hadi [9] studied the fluorescence spectrum for fluorescein solution in ethanol at different temperatures from room temperature to freezing point of solvent, using liquid nitrogen, and estimate theoretical model for this effect on fluorescence spectrum by using (Table curve 2D version 5.01) program. Also, the effect of temperature on fluorescence spectrum of fluorescein solution in methanol was studied experimentally and theoretically by M. F. Al- Kadhemy et al [10].

A variety of experimental techniques have been employed for understanding the lasing and photo physical properties (Rh6G) in solid host matrices [1,11]. The property of fluorescence can occur when particles return to ground level emitting photons as fluorescence 
spectrum is defined as the spontaneous emission process occurs between the two levels of the same pluralism [12]. At room temperature most of the molecules are in the lowest level of vibration ground level so the absorption occurs in the zero vibration ground level to a vibration level of excited state. The aim of this work is to study the effect of temperature on the fluorescence spectrum of Rhodamine $6 \mathrm{G}$ solution in acetone experimentally and theoretically.

\section{EXPERIMENTAL WORK}

The molecular formula for Rhodamine $6 \mathrm{G}$ is $\mathrm{C}_{28} \mathrm{H}_{31} \mathrm{~N}_{2} \mathrm{O}_{3} \mathrm{Cl}$, with molecular weight $479.02 \mathrm{~g} / \mathrm{mol}$ and its chemical structure is shown in Fig. (1) [13]. The molecular formula for acetone $\mathrm{C}_{3} \mathrm{H}_{6} \mathrm{O}$, molecular weight is $(58.08 \mathrm{~g} / \mathrm{mol})$. The dye solution is prepared according to equation below [14]

$$
\mathrm{m}=\mathrm{CVM} \mathrm{M}_{\mathrm{w}}
$$

where $\mathrm{m}$, and $\mathrm{M}_{\mathrm{w}}$ represent weight and molecular weight of Rh6G dye. C is concentration of dye solution, and $\mathrm{V}$ is the volume of solvent used. The concentration of dye solution is $1 \times 10^{-3}$ $\mathrm{mol} / \mathrm{liter}$. Liquid Nitrogen is nitrogen in a liquid state at an extremely low temperature used to decrease temperature of dye solution. Liquid nitrogen is a colorless clear liquid. At atmospheric pressure, liquid nitrogen boils at $-196^{\circ} \mathrm{C}(77 \mathrm{~K} ;-321 \mathrm{~F})$ and is a cryogenic fluid that can cause rapid freezing on contact with living tissue. Liquid nitrogen can easily be converted to a solid by placing it in a vacuum chamber pumped by a rotary vacuum pump [15]. Liquid nitrogen freezes at $63 \mathrm{~K}\left(-210^{\circ} \mathrm{C} ;-346 \mathrm{~F}\right)$. So that liquid nitrogen was used to convert dye solution to solid state. The temperatures are used from room temperature $\left(26^{\circ} \mathrm{C}\right.$; $299 \mathrm{~K})$, to low temperatures $\left(-50{ }^{\circ} \mathrm{C} ; 223 \mathrm{~K}\right),\left(-80{ }^{\circ} \mathrm{C}, 193 \mathrm{~K}\right)$, and $\left(-100{ }^{\circ} \mathrm{C} ; 173 \mathrm{~K}\right)$.<smiles>CCNc1cc2oc3cc(=[NH+]CC)c(C)cc-3c(-c3ccccc3C(=O)OCC)c2cc1C</smiles>

Figure 1. The chemical structure of Rhodamine 6G.

The fluorescence spectrum which emitted from the samples recorded by using fluorescence spectrofluorometer type (Jerrelash grating 82-410, 0.25 meter grating monochrometer, and with fluorescence monochrometer 75-1000, 1 meter Gzenny - Turner spectrometer spectrograph). 


\section{RESULTS AND DISCUSSIONS}

\section{1. Experimental part}

The fluorescence spectrum of Rhodamine 6G (R6G) in acetone on different temperatures from room temperature to nearly freezing point of liquid nitrogen as; $(T=299$, 223, 193, and $173 \mathrm{~K}$ ) were studied. We noticed that the intensity of fluorescence spectrum decreases with increasing temperature and fluorescence spectrum become more extensive as we move away from the room temperature as shown in Fig. (2), this means the fluorescence spectrum undergoes significant changes with temperature changes [16]. When temperature increases, the vibration and rotational energies increase as a result of thermal excitation led to broad spectrum. But when temperature decreases, the broad band transfer to sharp band because of low thermal excitation, and emission lines arise from one or most of rotational levels, where the spectrum resulting from higher levels disappear due to decreasing number of atoms in these levels.

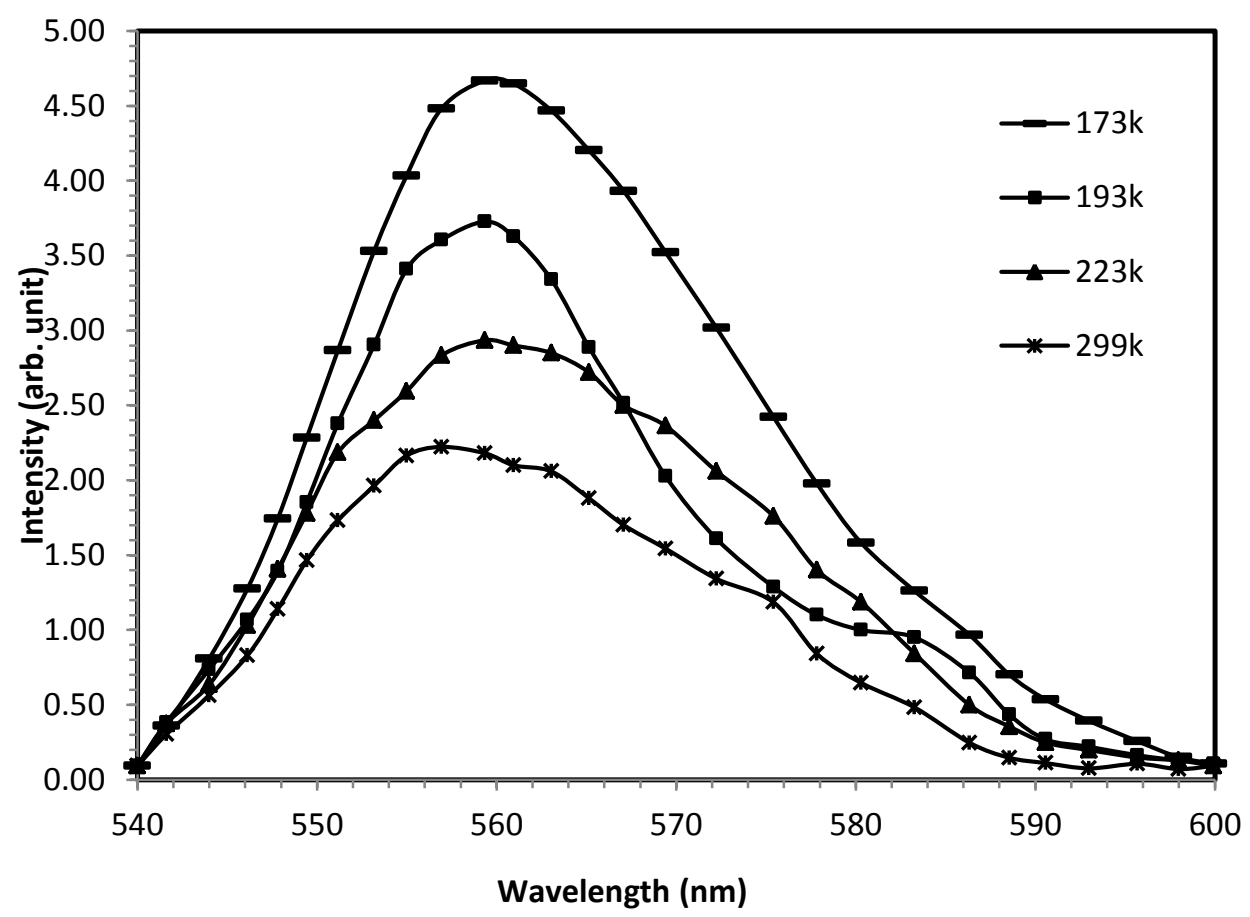

Figure 2. Fluorescence spectrum of (Rh6G) in acetone at different temperatures.

\section{2. Theoretical part}

For the importance of the effect of temperature on fluorescence spectrum of laser dye Rh6G; so that the development of theoretical model for this effect was very useful. The theoretical model was estimated by making curve fitting for all fluorescence spectrum of (Rh6G) in acetone in Fig. (2), and the resulted for each case are shown in Figs. (3; a, b, c, and d). Where the theoretical equation used was ExtraVal equation

$$
y=\operatorname{aexp}\left[-\exp \left(-\left(\frac{x-b}{c}\right)\right)-\left(\frac{x-b}{c}\right)+1\right]
$$




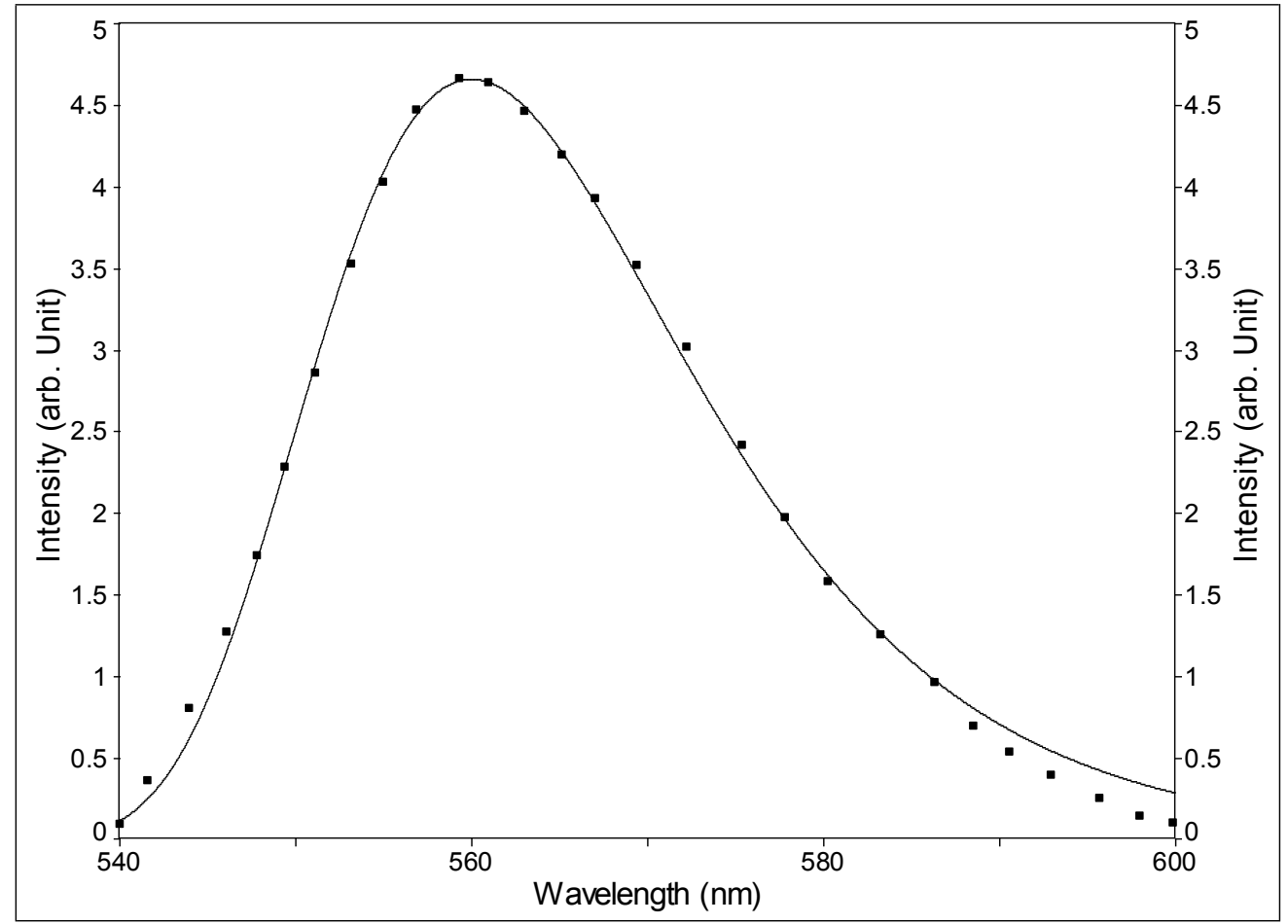

(a)

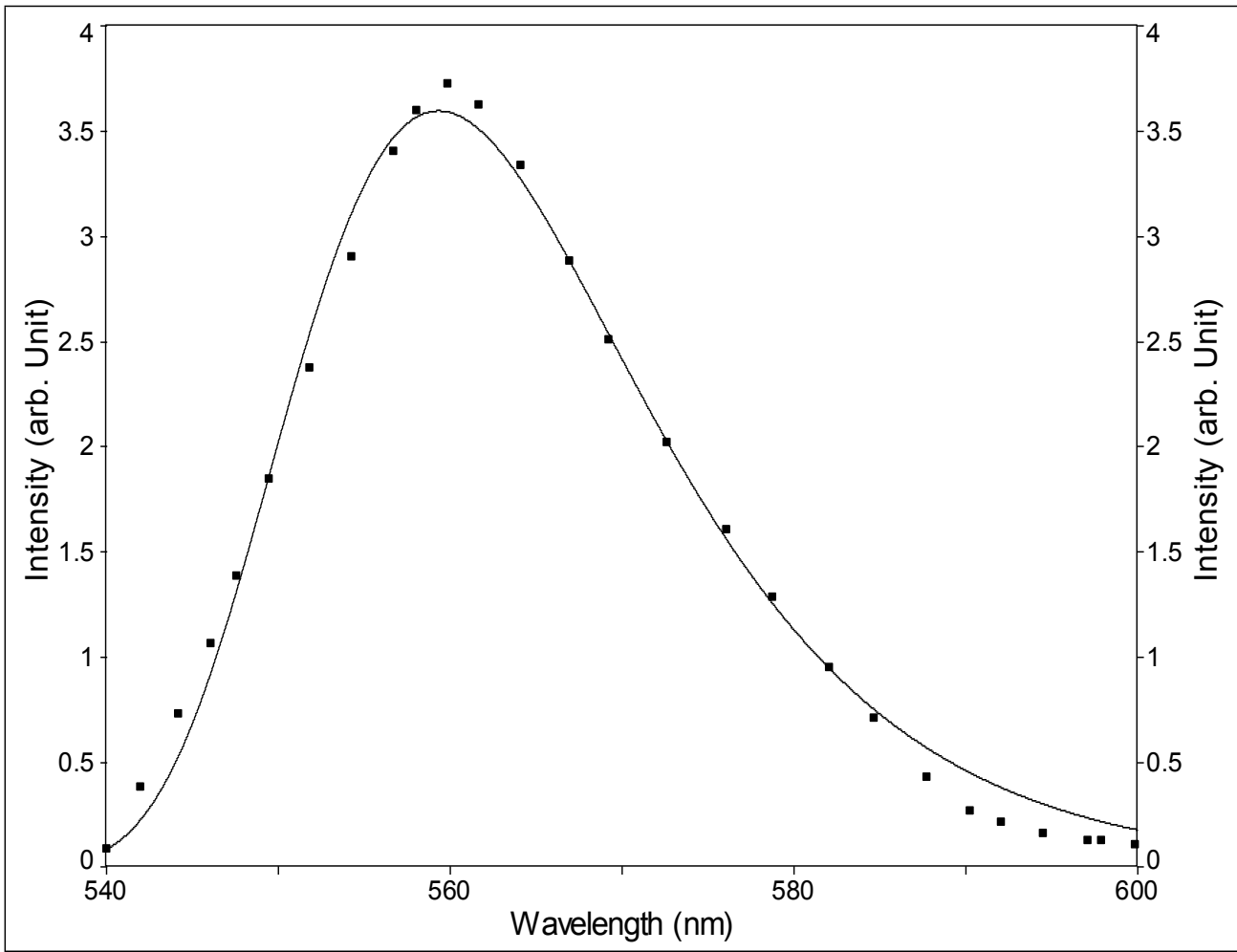

(b) 


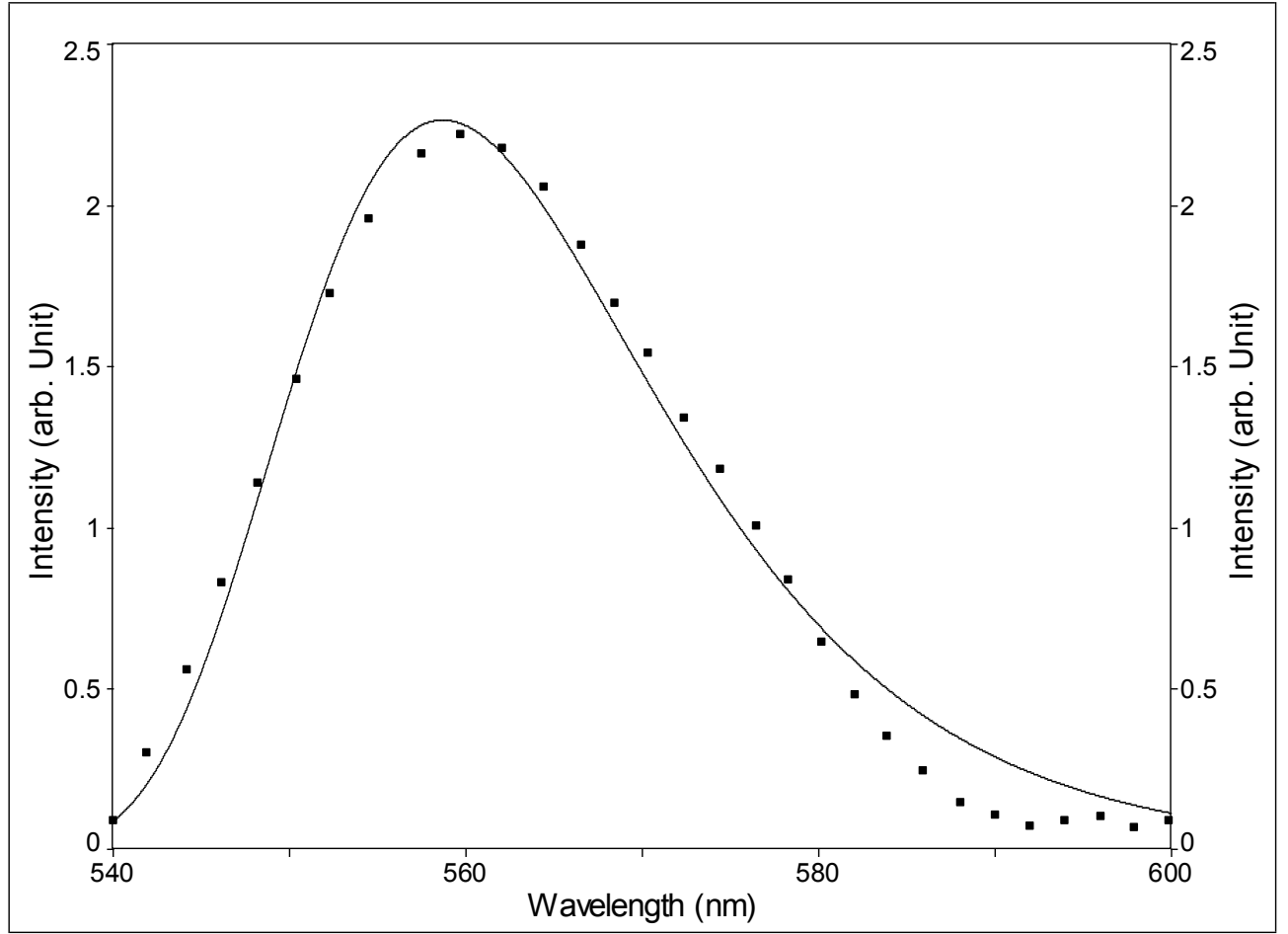

(c)

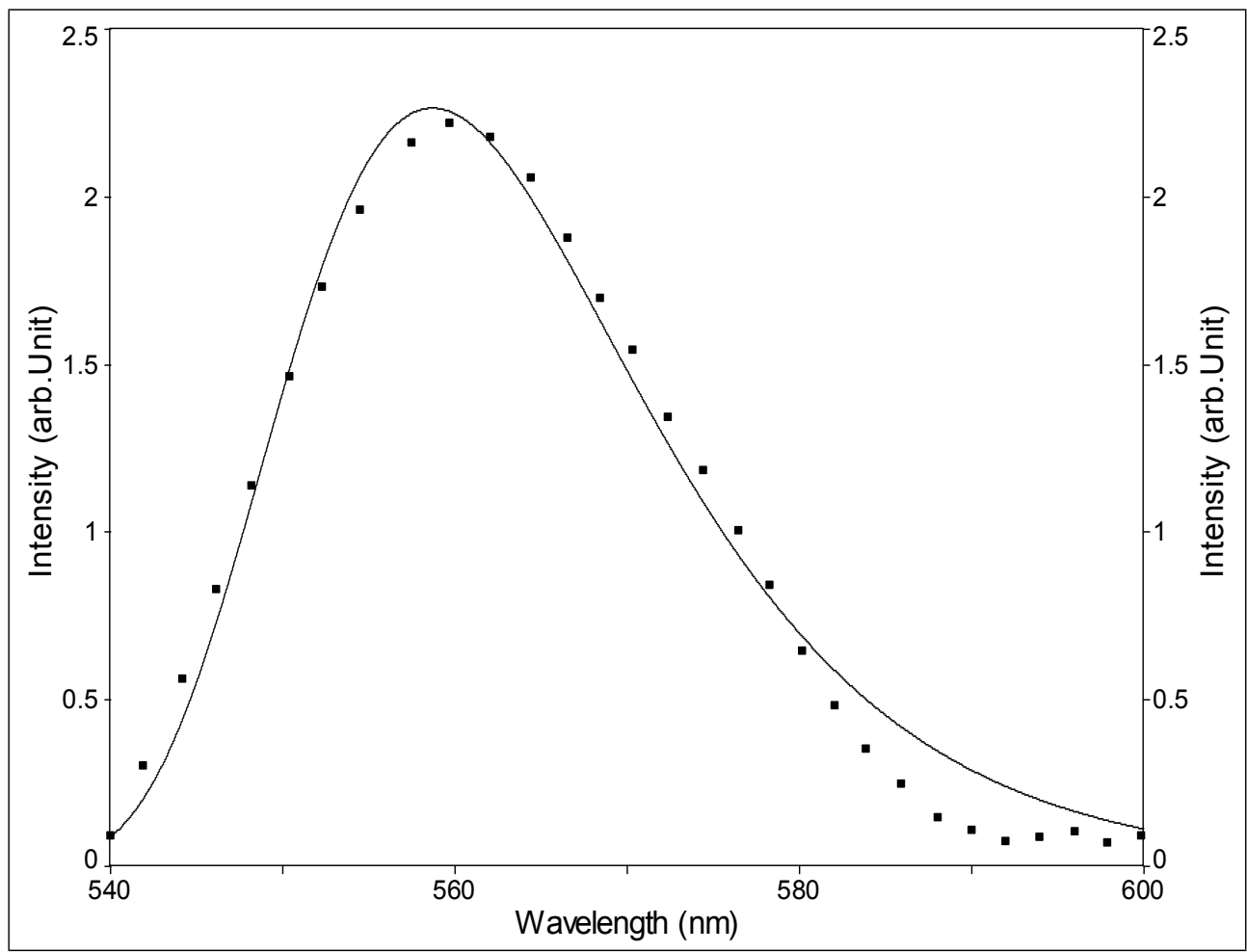

(d)

Figure 3. Fitting curve for fluorescence spectrum of (R6G) in acetone at (a) $\mathrm{T}=173$, (b) $\mathrm{T}=193$, (c) $\mathrm{T}=223 \mathrm{~K}$, (d) $\mathrm{T}=299 \mathrm{~K}$. 
where: $x$ and y represent wavelength and intensity of fluorescence spectra, respectively. The values of parameters $a, b$, and $c$ are shown in Table (1) for each temperature. Also, the correlation factor $r^{2}$ between experimental and theoretical data were illustrated in this table.

Table 1. The parameters of Extra Val equation for each.

\begin{tabular}{|c|c|c|c|c|}
\hline Parameter & $\mathbf{T}=\mathbf{1 7 3} \mathbf{K}$ & $\mathbf{T}=\mathbf{1 9 3} \mathbf{K}$ & $\mathbf{T}=\mathbf{2 2 3 K}$ & $\mathbf{T}=\mathbf{2 9 9} \mathbf{K}$ \\
\hline $\mathbf{a}$ & 4.6637508 & 3.5987861 & 2.9601147 & 2.266619 \\
\hline $\mathbf{b}$ & 559.95827 & 559.28792 & 559.24376 & 558.66402 \\
\hline $\mathbf{c}$ & 10.614296 & 10.211776 & 10.764065 & 10.400545 \\
\hline $\mathbf{r}^{\mathbf{2}}$ & 0.9966158609 & 0.99253793 & 0.99071244 & 0.98348598 \\
\hline
\end{tabular}

Figures (4-6) describe the variation of each parameter with temperature, and we take fitting curve for each one, the fitting equation being illustrated above the curves by using different fitting equations for these parameters. $\mathrm{y}_{1}, \mathrm{y}_{2}$, and $\mathrm{y}_{3}$ represent $\mathrm{a}, \mathrm{b}$, and $\mathrm{c}$ parameter, respectively. $\mathrm{x}_{1}$ acts temperature in figures and fitting equations.

$$
Y_{1}^{-1}=0.451468715+4.79626 e-5 x_{1} \ln x_{1}-8364.10105 / x_{1}{ }^{2}
$$

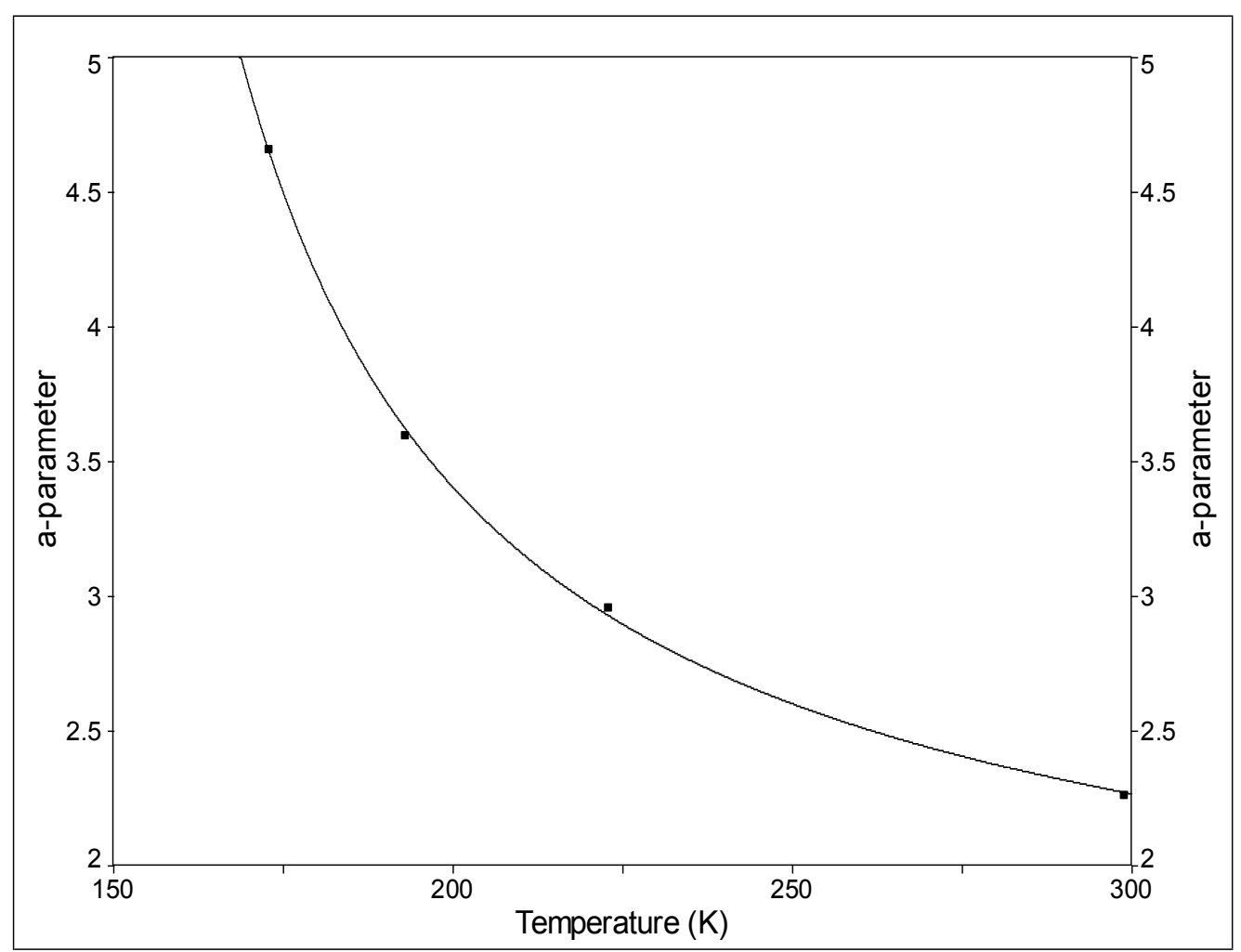

Figure 4. The relation between a-parameter and temperature. 


$$
Y_{2}=\frac{\left(557.92686-115.19377 \ln x_{1}\right)}{\left(1-0.20642326 \ln x_{1}\right)}
$$

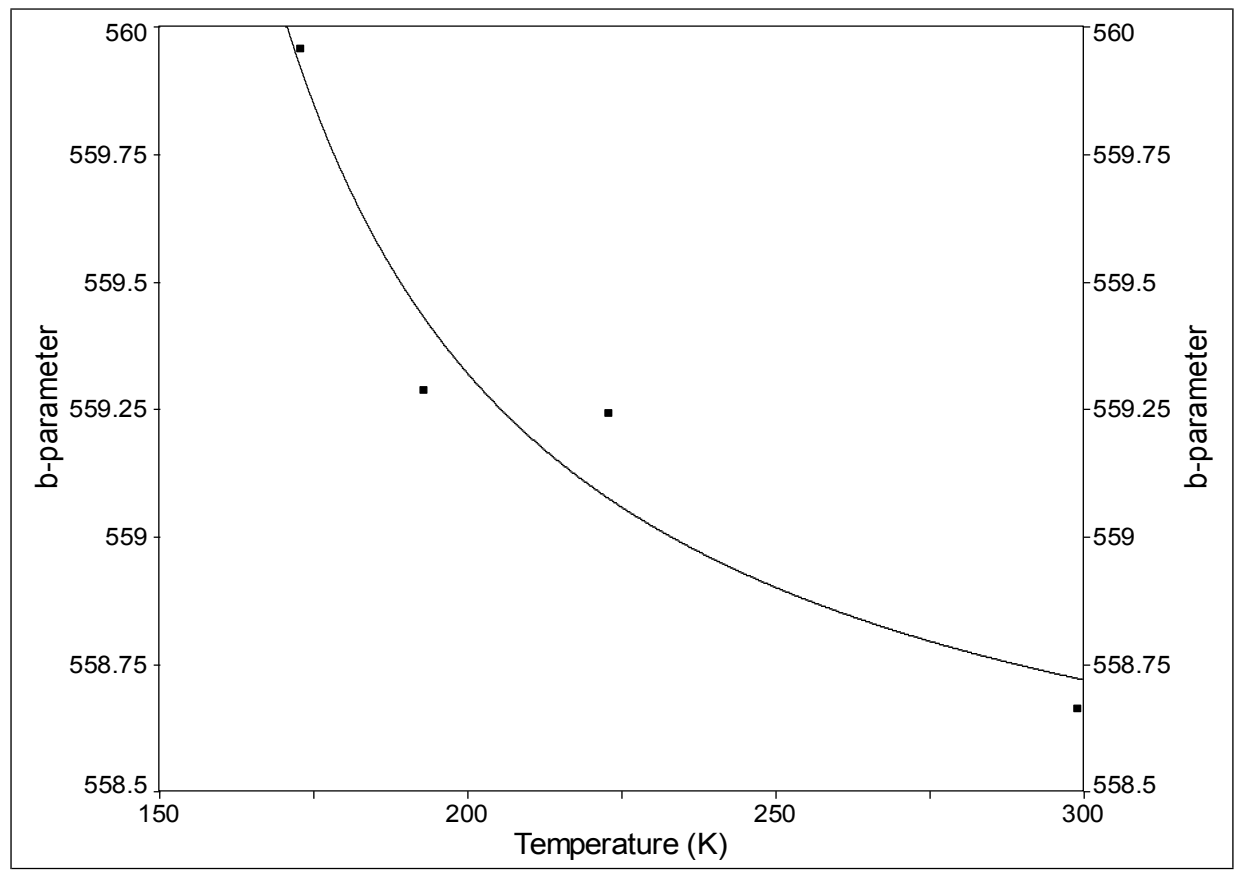

Figure 5. The relation between b-parameter and temperature.

$$
Y_{3}=9.8417809+2.6887987 e-5 x_{1}{ }^{2}-2.2932832 e-10 x_{1}{ }^{4}
$$

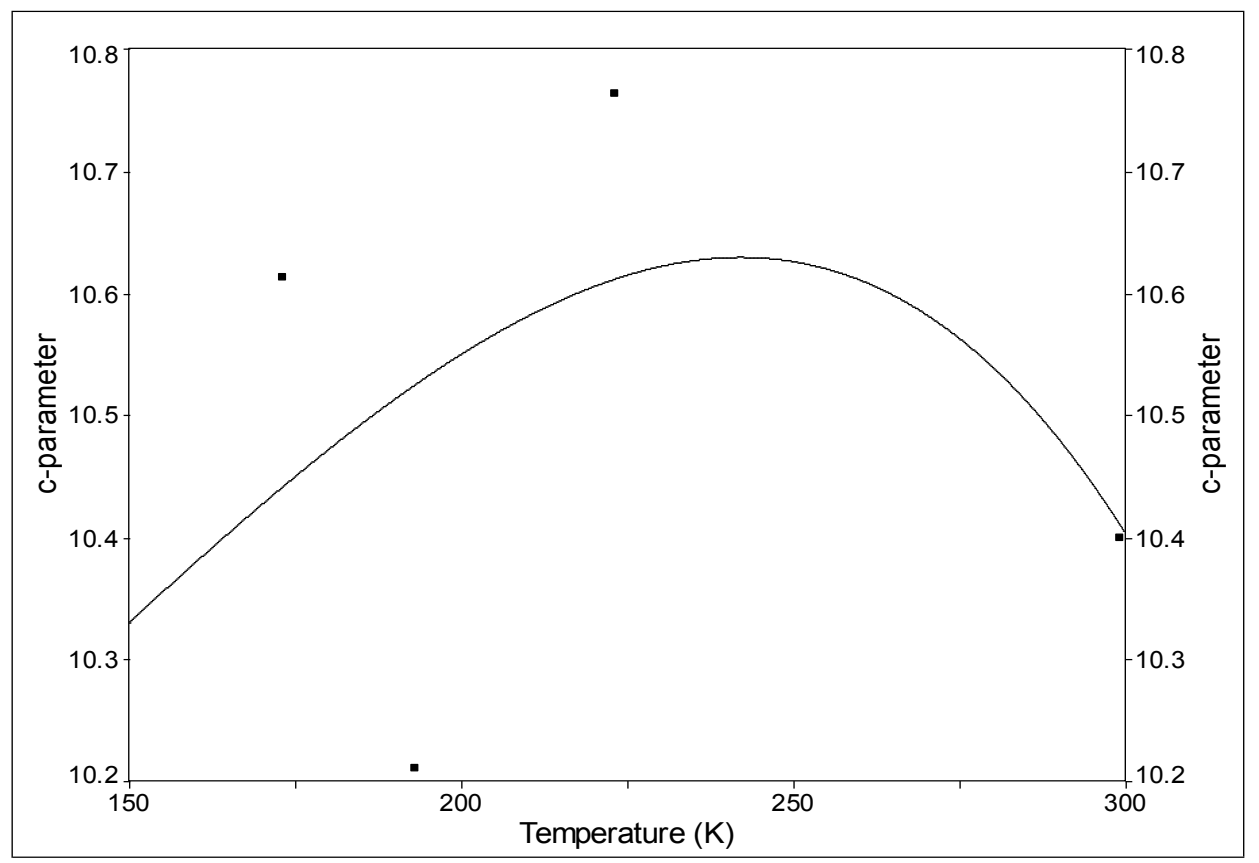

Figure 6. The relation between c-parameter and temperature. 
We calculated the final parameters from above fitting equations as test to find the theoretical equation for $(\mathrm{Rh} 6 \mathrm{G})$ in acetone spectrum at $\mathrm{T}=210$ and $250 \mathrm{~K}$, the estimated equation as follow:

Test Temperature $210 \mathrm{~K}$

$\mathrm{Y}=3.1679313 * \operatorname{EXP}(-\mathrm{EXP}(-((\mathrm{X}-559.19921) / 10.38154))-((\mathrm{X}-559.19921) / 10.38159)+1)$

Test Temperature $250 \mathrm{~K}$

$\mathrm{Y}=2.605191 * \operatorname{EXP}(-\mathrm{EXP}(-((\mathrm{X}-558.90236) / 10.626466))-((\mathrm{X}-558.90236) / 10.626466)+1)$

Note from Figure (7) that, when compared the results from this study with practical results at temperatures $(\mathrm{T}=210$ and $225 \mathrm{~K})$ that theoretical model gave results approximating somewhat to practical results and that means it can use this model to study fluorescence spectrum at any temperature we need without returning to study it practically.

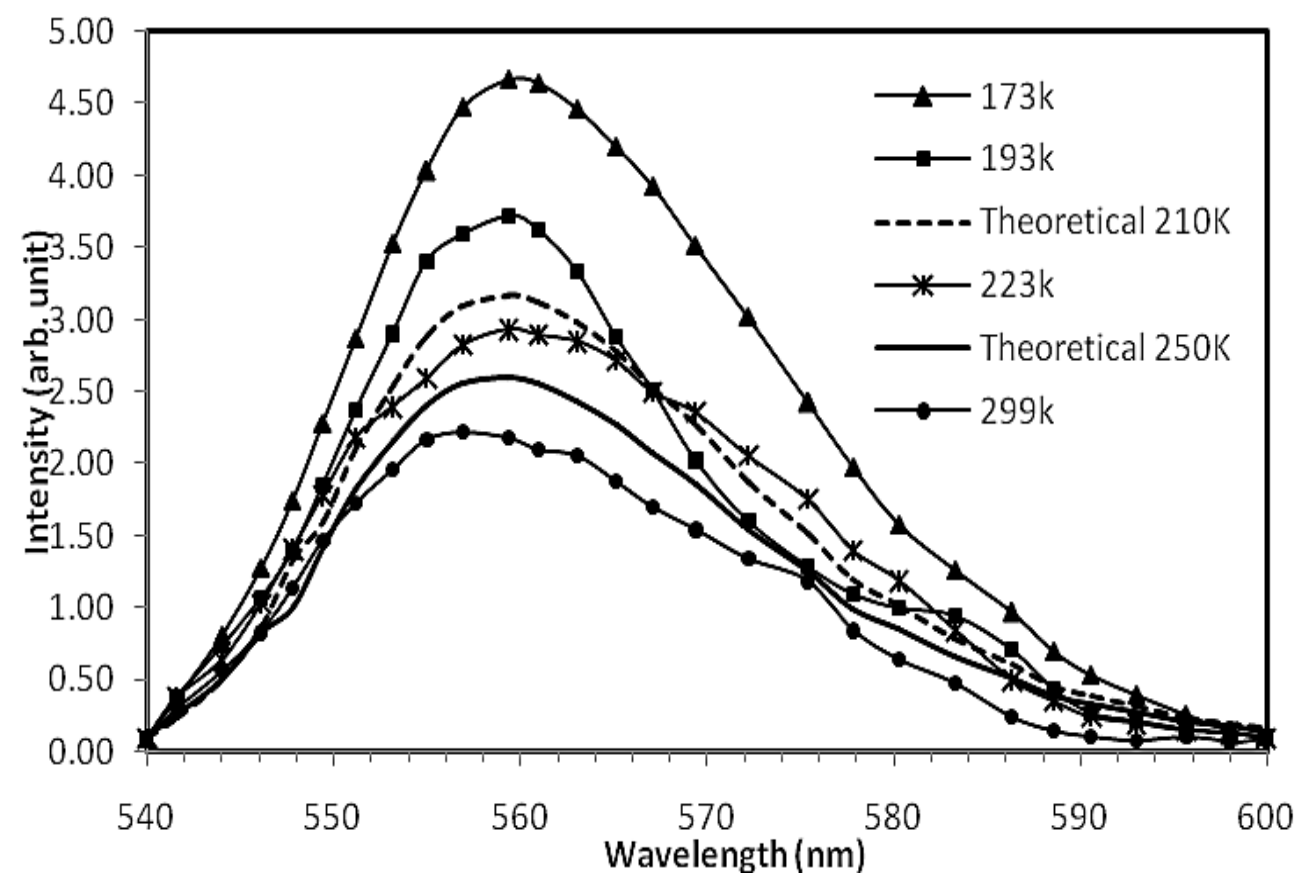

Figure 7. Theoretical and experimental fluorescence spectrum of $(\mathrm{Rh} 6 \mathrm{G})$ in acetone at $\mathrm{T}=210$ and $250 \mathrm{~K}$.

\section{CONCLUSION}

There is significant change in intensity for fluorescence spectrum of Rhodamine 6G solution in acetone from room temperature to freezing point of solvent by using liquid nitrogen; this is interest for improving operation of dye laser. Estimated theoretical model for this effect benefit and led to get fluorescence spectrum of Rh $6 \mathrm{G}$ at any temperature without practical test. 


\section{References}

[1] P. Boutin, J. Mugnier, B. Valeur, J. Fluorescence 7 (1997) 215S-218S.

[2] S. Venugopal Rao, N. K. M. Naga Srinivas, D. Narayana Rao, Chemical physics letters 361(5-6) (2002) 439-445.

[3] S. Haghighat, D. J. Castro, R.B. Lufkin, H. R.Fetterman, D. J. Castro, J. Soudant, P.H. Ward, R.E. Saxton, Laryngoscope 102 (1992) 81.

[4] M. J. Blackman, J. E. T. Corrie, J. C. Croney, G. Kelly, J. F. Eccleston, D. M. Jameson, Biochemistry 41 (2002) 12244-12252.

[5] J. R. Lakowicz, Principles of fluorescence spectroscopy, third edition, Plenum Press, New York, 1968.

[6] M. U. Belyi and A. B. Leontev, Opt. Spektrosc. 34 (1972) 715-721.

[7] F. P. Schafer, Dye Lasers, Second Revised Edition, Springer-Verlag, Berlin, Heidelberg, New York, 1977.

[8] M. A. Ali, J. Moghaddasi, S. A. Ahmed, Laser Chem. 11 (1991) 31-38.

[9] M. F. Hadi, Atti Della, Fondazione Giorgio Ronchi 66 (2) (2011) 217-224.

[10] M. F. Hadi Al-Kadhemy, A. A. Dawood Al-Zuky, Kh. N. Abbas, Atti Della Fondazione Giorgio Ronchi 6 (2012) 819-828.

[11] G. Hungerford, K. Suhling, J.A. Ferreira, J. Photochem. Photobiol. A 129 (1999) 71.

[12] C. N. Banwell "Fundamental of Molecular Spectroscopy". $3^{\text {rd }}$ edition, May, 1983.

[13] A. M. Helmenstine, Chemical Structures Starting with the Letter R. About.com.chemistry. 2013.

[14] M. F. Hadi Al-Kadhemy, "Spectral properties of some organic dyes used as active media for dye lasers", MSC. Thesis in Baghdad Univ., 1995.

[15] W. Umrath, Journal of Microscopy 101 (1974) 103-105.

[16] I. Lopez Arbeloa, J. Photochem. 18 (1982) 161. 